\title{
Communicating with Humans and Robots: A Motion Tracking Data Glove for Enhanced Support of Deafblind
}

\author{
Federico Gelsomini ${ }^{1}$, Elena Tomasuolo ${ }^{2}$, Maria Roccaforte ${ }^{1}$, Patrick C. K. Hung ${ }^{3}$, Bill Kapralos ${ }^{3}$, Adam \\ Doubrowski $^{3}$, Alvaro Quevedo ${ }^{3}$, Kamen Kanev $^{4}$, Makoto Hosoda ${ }^{4}$, Hidenori Mimura ${ }^{4}$ \\ ${ }^{1}$ Sapienza Università di Roma, Rome, Italy \\ ${ }^{2}$ Istituto di Scienze e Tecnologie della Cognizione - CNR, Rome, Italy \\ ${ }^{3}$ Ontario Tech University, Oshawa, Canada \\ ${ }^{4}$ Shizuoka University, Hamamatsu, Japan
}

\begin{abstract}
In this work, we discuss the design and development of a communication system for enhanced support of the deafblind. The system is based on an advanced motion tracking Data Glove that allows for high fidelity determination of finger postures with consequent identification of the basic Malossi alphabet signs. A natural, easy-to-master alphabet extension that supports single-hand signing without touch surface sensing is described, and different scenarios for its use are discussed. The focus is on using the extended Malossi alphabet as a communication medium in a Data Glove-based interface for remote messaging and interactive control of mobile robots. This may be of particular interest to the deafblind community, where distant communications and robotized support and services are rising. The designed Data Glove-based communication interface requires minimal adjustments to the Malossi alphabet and can be mastered after a short training period. The natural interaction style supported by the Data Glove and the popularity of the Malossi alphabet among the deafblind should greatly facilitate the wider adoption of the developed interface.
\end{abstract}

\section{Introduction}

Deafblindness has been defined as a sensory disability that includes a combination of visual and hearing impairment in the same individual [1]. Deafblindness can be acquired after the development of language (this is known as post-lingual deafblindness), or an individual may be born both deaf and blind, or become deaf and blind early in life prior to the development of language (pre-lingual deafblindness) [2]. Individuals with congenital deafblindness must develop language and the ability to communicate without vision and hearing. In contrast, people with acquired deafblindness must maintain language and the ability to communicate when deprived of previously good hearing and/or vision [2]. It is estimated that approximately 1.5 million people globally are completely deafblind, compared to 150 million people with different levels of blindness associated with deafness [3]. Given the importance placed on vision and hearing in our daily activities, including communication [4], accessing information, and mobility, those with deafblindness face many complex challenges that may hinder their functioning and participation in daily events and activities and require a variety of assistive, educational, and therapeutic services [5].

As Olson et al. point out, the convergence of technologies such as sensors and signal processing, machine learning, data and image processing smart and haptic interfaces, and the growing interest and application of the Internet of Things (IoT), has led to new modes of connectivity and communication [4]. This in turn is providing the potential to overcome many challenges for those who have traditionally faced barriers to free communications due to circumstances associated with deafblindness. Communication based on the sense of touch (i.e., tactile-based) is a fundamental aspect of social life for deafblind people who heavily rely on this given their inability to communicate using audio and visual cues [6]. The recent advancements in haptic (machine touch and human-machine touch interactions) technologies provide us with opportunities to develop innovative assistive technologies for the deafblind. However, the availability of technology is not sufficient; adequate communication solutions remain minimal, and new intelligent, multimodal, personalized communication interfaces are required to meet the needs of the deafblind [4].

In addition to what deafblind people can do to improve their communication level, it is important to remember that the surrounding environment and society play a fundamental role in improving the living conditions of individuals with sensory deficits. This materializes in new inclusive policies to remove 
physical and social barriers and provide greater aids and assistive services to the deafblind. Progress in science and technology plays an important role in implementing such policies by providing facilities for studies and detailed analysis of the corresponding needs followed by the creation of software, hardware, and technological solutions that meet the specific needs of deafblind individuals. In particular, such technology-assisted interactions could provide the deafblind community with a functional communication base in the absence of a caretaker and/or in social distancing or isolation situations as imposed by the recent COVID-19 pandemic [7].

In the following sections of this work, we review several communication methods commonly used by the deafblind and discuss the technological enhancements to facilitate the integration into a contemporary social environment. The final section is dedicated to specialized equipment and software designed for hand interactions employing an extension of the Malossi alphabet.

\section{Deaf-blind communication methods and related technological enhancements}

Various modes of communication have been devised and used over time, addressing behavioral, pictographic, and object communication [8]. With respect to deafblind people, the most effective methods for establishing relations and sharing meanings with others are those that implement functional communication through touch and gestures [9]. For example, congenitally deaf people who have lost their sight at some point in their lives will primarily use sign language while those born blind with acquired deafness later in their life are more likely to use a tactile language. In general, the communication systems commonly used by deafblind people favor touch as the main sensory channel; these include real languages, such as tactile sign languages, different deafblind alphabets, and systems based on the literacy skills of the vocal language known by the deafblind person, including dactylology. Alternative communication approaches employing natural interfaces based on behavioral, object, and pictographic communication are also available, although they are usually confined to a more limited range of content. A summary of the most common touch-based communication methods for the deafblind, along with a discussion of possible extensions and technological enhancements for communication support, are presented below. With respect to written language support, we briefly discuss Braille, which is the de facto standard for the deafblind community.

\subsection{Tactile Sign Language (TLS)}

Tactile sign language (TLS) is a form of sign language employed by deafblind individuals whereby a signer follows a conversation by using one or both of their hands to perceive, through touch, the signs being produced [10]. As a sign language, TLS requires the movement of the hand and arms. Therefore, it lends itself to 3D hand tracking technology, with the possibility of rendering both the hand shape formation and their movement in the three-dimensional space between the users. While this form of augmentation can be of great importance for the rapid digital conversion of messages, it is mostly applicable to message delivery and will have a limited impact on message reception. TLS can also be used to facilitate dactylology ("finger spelling"), that is, the technique of using signs made with the fingers to "spell" certain words. Conversely, piezoelectric or magnetic vibrators can be attached to a wearable interface and used to support the reception of messages in the form of vibrational stimuli.

\subsection{Malossi Alphabet}

The Malossi alphabet is a method of communication whereby the hand (usually the left hand) becomes a typewriter for the receiver of the message; the sender composes words letter by letter by touching and pinching different parts of the interlocutor's palm in a sequence that corresponds to the communicated characters [8]. The Malossi method is often used by individuals who previously learned to read and write before becoming deafblind and is taught to children. The interlocutor pushes upon or pinches the deafblind person's hand at the places denoted by letters of the alphabet (Figure 1), providing the possibility to form words and sentences. More specifically, the letters from "A" to "O" are pressed while the letters from "P" to "Z" are pinched. Numbers are expressed as in the Braille alphabet, namely using the first 10 alphabet characters, preceded by the letter "N").

This communication method can be technologyenhanced through palm-based pressure sensors positioned in the spots corresponding to the Malossi alphabet letters for the outgoing messages. Further haptic feedback for the incoming messages could be provided through vibrational elements placed on the back of the user's hand. An improved version of such an interface can employ more advanced piezoelectric components that act as both pressure sensors and vibrators. In this case both the input and the output interface components could be placed entirely on the palm of the user's hand. 


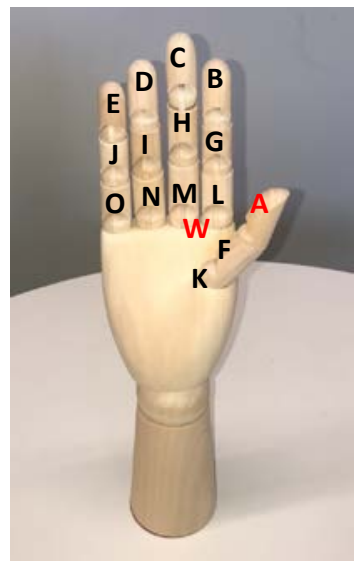

(a)

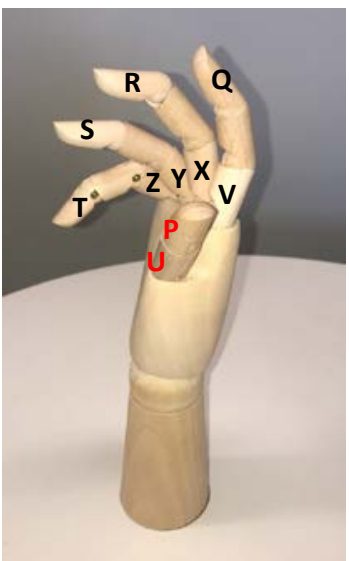

(b)
Figure 1. The Malossi alphabet with the pressed (a) and the pinched (b) letters ( $\mathrm{W}$ is pinched but seen better in left view)

\subsection{Lorm Alphabet}

The Lorm alphabet is a tactile alphabet developed in the late $19^{\text {th }}$ century by Hieronymus Lorm [11] that allows for interactions based on characteristic gestures such as single and multiple touches, squeezes, and tracing of linear and circular shapes on the palm or the fingers of the receiver's hand. The vowels A, E, I, O, and $U$ are communicated by a single touch to the tip of the corresponding finger while a linear motion from the tip to the bottom of the corresponding finger communicates the consonants B, D, G, and $\mathrm{H}$. Other letters require simultaneous touches with more than one finger, e.g., two for $\mathrm{N}$ and $\mathrm{W}$, three for $\mathrm{M}$, and four for K. Some letters require more complex actions such as drumming with several fingers on the palm for R, squeezing together the tips of the index and the middle fingers for F, drawing a circular shape in the middle of the palm for $\mathrm{S}$, and so on.

This communication method can also benefit from technological enhancements similar to the ones applicable to the Malossi alphabet. However further enhancements are required to support the more complex gestures, particularly the squeezing and the linear and circular motions. This could be implemented, for example, with a flexible touch-sensitive surface (a flexible tablet) embedded in a glove with integrated actuators for haptic feedback.

\subsection{Deafblind Manual Alphabet}

The deafblind manual alphabet constitutes an adapted form of finger spelling taken from the British Sign Language (BSL). This method implements gesture interactions through touching of the palm and the fingers and movements onto the palm and between the fingers. Since it also involves finger grasping, curling, and fist clenching and uses the outer part of the palm, adequate technological augmentations cannot be achieved by simply combining the approaches presented so far. Indeed, a specialized wearable interface such as a Data Glove is needed for tracking the complex motions of the user's hands following recognition of the three-dimensional gesture components for the outgoing messages. In addition, haptic feedback as discussed in the Malossi and Lorm alphabet sections, could be used for the incoming messages.

\subsection{Spartan Block Alphabet}

The Spartan block alphabet is another form of alphabet-based manual communication implemented by writing capital letters on the palm of the user's hand. The interlocutor will use their fingers to write the capital letters of the word to be communicated. In this case, the possible technological augmentations are similar to those used in dactylology, e.g., the Lorm alpahabet method. The Spartan block alphabet is entirely based on simple lines and curves that can be tracked by a flexible touch sensitive surface.

\subsection{Tadoma}

The Tadoma method implements another form of manual communication that is not based on an alphabet. With the Tandoma method, hands are placed on the cheeks and thumbs on the mouth of the interlocutor, for tactile sensing and understanding of the spoken words. As the Tadoma method is strictly related to the speaker's mouth and jaw, a technological augmentation should focus on tracking the lip's motions and sound vibrations. This could be achieved with an augmented version of a Data Glove-based interface with embedded pressure sensors. Such an augmentation would be beneficial in distant communication setouts and/or in cases where direct physical contact must be avoided.

\subsection{Object Assisted Communication}

The object-assisted communication method for the deafblind employs different physical objects as representations of notions and activities. Such objects must be recognizable, meaningful, and maintain good tactile similarity with what they represent [12]. For example, a small glass can be used by a deafblind person to express their need to drink. This communication method is usually used by people with 
intellectual disabilities and limited visual residue.

In our previous works [13, 14], we employed surface-based interactions to support collaborative learning and augmented reading through mobile devices that react to events triggered by digitally encoded surfaces. Similarly, augmented actions such as sending messages to distant caretakers while providing dedicated feedback to the deafblind individual can be triggered by interacting with the object, as shown in Figure 2.

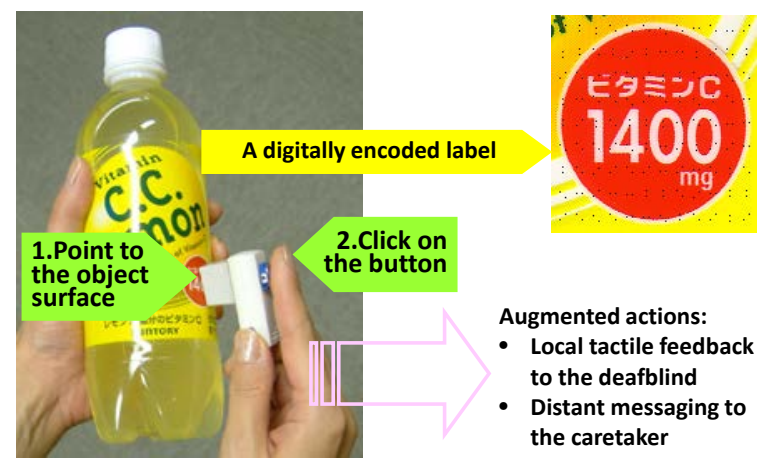

Figure 2. Object assisted communication

\subsection{Braille}

In contrast to the deafblind communication methods presented so far, Braille represents a written language that is less used in interactive setups. As a reading and writing method, it is primarily used by people with congenital blindness and acquired deafness [15]. The characters of Braille constitute dot patterns that are suitable for tactile sensing when embossed on thick paper sheets (see [16] for greater details). As machine translation into Braille is quite common nowadays, it can be used for enhancing the more interactive deafblind communication methods presented earlier. In particular, wearable interfaces could be programmed to provide haptic feedback that simulates the sensations of touching and scanning Braille scripts.

\section{Glove-assisted communication systems for the deafblind}

A diverse array of communication assistive systems employing some form of technologically augmented glove have been developed and experimented with over the years [17, 18, 19, 20, 21]. Common to this prior work is some form of hand/touch-based communication systems such as the Malossi, and Lorm deafblind alphabets, or Braille, and to a message sending strategy actuated by touching the palm, in correspondence with actuators situated on some sort of wearable support or glove. Message reception functions oppositely, having words and sentences coded as sequences of vibrational patterns on the palm and/or the back of the user's hand.

\subsection{The DB-HAND and dbGLOVE Systems}

The design and development of the DB-HAND system constitute an important step towards the realization of an assistive hardware and software system for the support of the deafblind [8]. It is implemented as a wearable glove-shaped communication device with touch sensors on its surface and embedded vibrational actuators for haptic feedback. The DB-HAND system supports input and output based on the Malossi alphabet, and therefore all of the sensors and actuators are positioned in correspondence with the Malossi language touchpoints locations. Deafblind users send messages through tactile impulses effectuated by one of their hands positioned over the transducers on the palm of the other, glove-wearing hand. More recently, a more advanced dBGLOVE system with multilingual support was designed [3]. It enables deafblind users to choose their preferred tactile language among those supported by the system. In addition, it aims to improve touchenabled bi-directional text-based communication by adding visualization on portable devices screens, translations, and voice conversion.

\subsection{The Mobile Lorm Glove and the GlovePi and GLOS Systems}

Other applications have employed gloves similar to the DB-HAND system while utilizing the Lorm alphabet. The Mobile Lorm Glove [22] has been designed to handle the different palm-based Lorm gestures instead of the pressing and pinching actions required by Malossi. On the technical side, the Mobile Lorm Glove is equipped with flat piezoresistive fabric sensors that change electrical resistance when pressed to avoid interference with gesture flow, differently from the DB-HAND system pressure sensors that stick out of the glove. The output unit is realized with coinshaped vibrating motors, placed on the back of the hand to translate incoming messages into Lorm alphabet patterns

Following the high popularity and penetration rate of mobile devices and open source solutions, some low-cost communication systems to support deafblind individuals have also been developed. The GlovePi [23] is a Malossi alphabet modular solution based on a glove equipped with push buttons on the palm for the letters from $\mathrm{A}$ to $\mathrm{O}$. In addition it uses conductive 
silver pads on the back of the hand for the letters from $\mathrm{P}$ to Z.

\subsection{Limitations of the existing systems and possible technological enhancements}

While the deafblind assistive methods and systems discussed so far have great potential, they also have various limitations. The DB-HAND system, for example, is rather bulky and thus not so comfortable to wear and use. The Lorm Glove may appear more compelling but it relies on substantial modifications of the traditional Lorm alphabet and may thus require long-term training. The GlovePi is particularly attractive as a low-budget and quick access solution, but its daily usage can be quite challenging, and thus regular maintenance may be required.

The above mentioned issues can be addressed by a specialized system that employs hardware and software components specifically designed to meet the finger tracking requirements of the target Data Glove applications, as discussed in the following section.

\section{Specialized equipment and software for deafblind communications support}

The subtle finger motions of the human hand are difficult to track with traditional image-based motion capture techniques. One of the most widely used alternatives is to employ a more specialized device such as a Data Glove that is capable of detecting the bending angles of the finger joints when worn. Such Data Gloves are designed to digitize the motions of the human hands by tracking the changes in the electric signals produced by the sensors embedded in the device. While some commercial data gloves are advertised as providing a good fit to human hands, most of them feel hard and tight because of the bulky sensors and the corresponding protective fabric layers. The employed sensor technology ultimately determines the touch and feel of the Data Gloves and therefore, very thin elastic Data Gloves resembling, for example, surgical gloves, would be rather difficult to produce.

In the following sections, we will consider different technology-based solutions relying on recent advancements in nanotechnology and other advanced fields that could further facilitate and enhance the interaction capacity of the deafblind.

\subsection{Higher fidelity finger tracking with stretchable Carbon Nanotube (CNT) sensors}

Given the current state of the art, a light elastic Data Glove with a lower wearing burden has been developed by incorporating specialized carbon nanotube (CNT) strain sensors along with the finger joint lines on a surface of thin compression fabric gloves. Such gloves should be more suitable for longterm wear and continuous interactive communications by the deafblind in their everyday life. In the Data Glove, separate CNT strain sensors are provided for each Metacarpophalangeal (MP) joint and each Proximal Interphalangeal (PIP) joint of every finger to independently detect the degree of bending of each joint. When a finger joint bends, the respective CNT strain sensor elongates, and the resistance increases. When a finger joint is stretched, the sensor contracts and the resistance decreases.

In the developed stretchable sensors, millimeterlong multi-walled CNTs (MWCNTs) were unidirectionally aligned and sandwiched between elastomer layers [24]. The aligned CNT layer was formed by stacking CNT webs drawn from a spinnable CNT forest [25]. The measured sensor resistance is linearly proportional to the applied tensile strain; the higher the tensile strain, the higher the sensor resistance. The detection limit of this sensor is over $200 \%$ strain. The strain sensor shows that the resistance variation closely follows the temporal changes in the allied strain at a frequency of $29 \mathrm{~Hz}$, implying that the CNT strain sensor exhibits an excellent rapid response.

The present research devises a communications support circuit composed of three main elements: i) a data glove, ii) a processing unit, and iii) a mobile robot.

\subsection{Software and communications framework}

The Data Glove is capable of providing highfidelity feedback regarding the motions of the user's fingers and the palm of their hand. However, exercising different motions to generate experimental data while controlling the application responsible for the data gathering, maybe a difficult task given that the user will not be able to use their hands as usual. We have developed a framework for data glove-based finger posture motion tracking and recording with a simplified non-manual interface to alleviate this issue. The recorded postures and motion sequences are stored in the computer file system and can be retrieved and played as needed later.

The developed framework ensures that the user applications will interact with the real Data Glove and any simulated virtual data gloves similarly. We are providing a physical communication port connection that fully emulates the data glove and is practically indistinguishable from it when accessed by the application software. For this, the virtual Data Glove is implemented as a simulation software package that 
connects to the physical communication port, allowing the simulation and the application software to be run on either the same or different devices.

The physical Data Glove is an advanced device capable of tracking the subtle motions of the user's fingers in real-time. The analog data from the stretchable CNT sensors embedded in the fabric of the Data Glove is measured at a rate of approximately 250 times/sec. For this, the resistance of every sensor, which is proportional to its extension, is evaluated by measuring the incurred voltage drop. These measurements are conducted by a 14 bit analog to digital converter (ADC) and obtained digital voltage levels are stored in 22 byte long communication packages. The data glove application software communicates with the Data Glove at 400,000 baud, ensuring a package exchange rate of approximately 4 ms.

Wireless communication with the Data Glove through a StickPC has been successfully employed in both development and testing [26]. However, it would be much easier for the end-users to have a dedicated wireless box that connects to the data glove and works, rather than a fully-fledged Stick PC that requires continuous support and OS updates, etc. We have thus built a Data Glove WiFi communication box based on the XBee series of modules. Once paired, XBee modules provide a quick and easy, fully automated way of establishing a wireless serial connection. These connections can be directly used for information exchange both with computing devices and mobile robots [27].

\subsection{Integration of the Malossi alphabet}

The advanced CNT-based stretchable sensors embedded in the DataGlove ensure highly reliable tracking of the subtle motions of the user's fingers. It allows for more precise pose estimation for the recognitions of the Malossi alphabet signs at higher speeds. This constitutes a solid base for novel DataGlove-based applications of the Malossi alphabet in the digital world. Furthermore, we envisage different communications scenarios when the parties are not in direct physical contact to accommodate, for example, social distancing, and remote and mobile communications, etc.

In traditional Malossi alphabet, signing messages are received by sensing the touches on the recipient's hand. Supportive devices would therefore employ embedded pressure sensors to identify the touches and convert them to digital signals. However, the highfidelity finger motion tracking of the Data Glove allows for identifying such touches without additional pressure sensors. In this scenario, the touches are derived from finger motions and postures. There is no need for physical contact and therefore, non-contact communication becomes possible.

The approach outlined above can be extended to single hand signing in mobile setouts (refer to Figures 3 and 4) as follows:

- Pressed letters A-O, excluding A, F and $\mathrm{K}$ are signed by touching the designated spots with the tip of the thumb of the same hand (letters L, M, N, and $\mathrm{O}$ that require touches on the palm could be signed by touching the proximal phalange of the corresponding finger if easier to reach).

- Pressed letters $\mathrm{F}$ and $\mathrm{K}$ are signed by touching the designated spots with the tip of the index finger.

- Pinched letters Q, R, S, T, V, X, Y, and Z are signed by touching next to the designated spots on the side of the corresponding finger phalanges using the index finger.

- The remaining letters $\mathrm{A}, \mathrm{P}, \mathrm{U}$, and $\mathrm{W}$ are signed by touching with the thumb the side of the middle phalange of the index, middle, ring, and small fingers correspondingly.
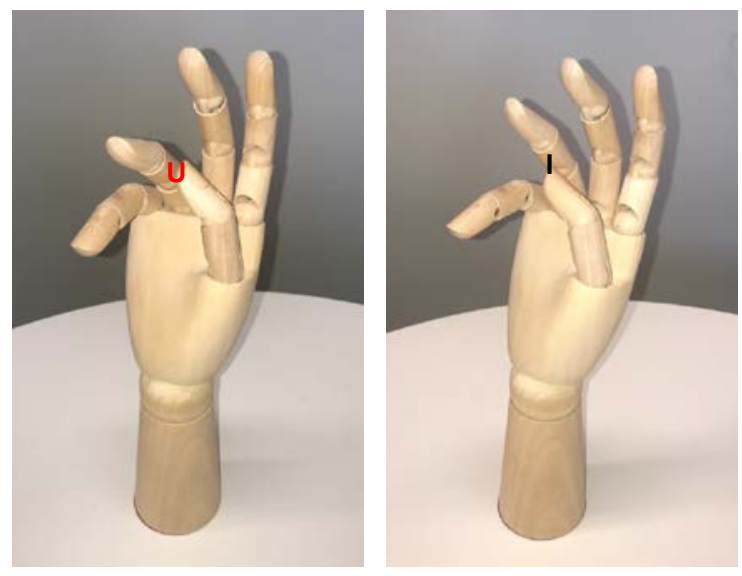

Figure 3. Single hand signing of U (left) and I (right)

The extension attempts to preserve the sensations of the Malossi alphabet letters as much as possible. Indeed, almost all pressed letters retain their original sensations as the pressure is exercised exactly on the same spots (Figure 4). This also applies to most of the pinched letters as they are signed by pressing the side of the finger phalanges where the original pinching takes place. Finally, the reallocation of the remaining four letters was done to the empty spots on the middle phalanges in a consistent and easy to remember way.

Moving the Malossi alphabet sign recognition from the receptive surface to the sender's hand opens a range of new possibilities. With the extended Malossi alphabet signing scheme, a deafblind person wearing a 
Data Glove can send messages simultaneously, even while in motion. Such messages can be voiced or visualized on a portable screen if, for example, a deafblind person needs immediate assistance in the street. Messages can also be copied to an assistance center or transmitted to an emergency service as needed. Messages in truly electronic format can also be used directly, e.g., to communicate with and control a mobile robot serving as a guide.
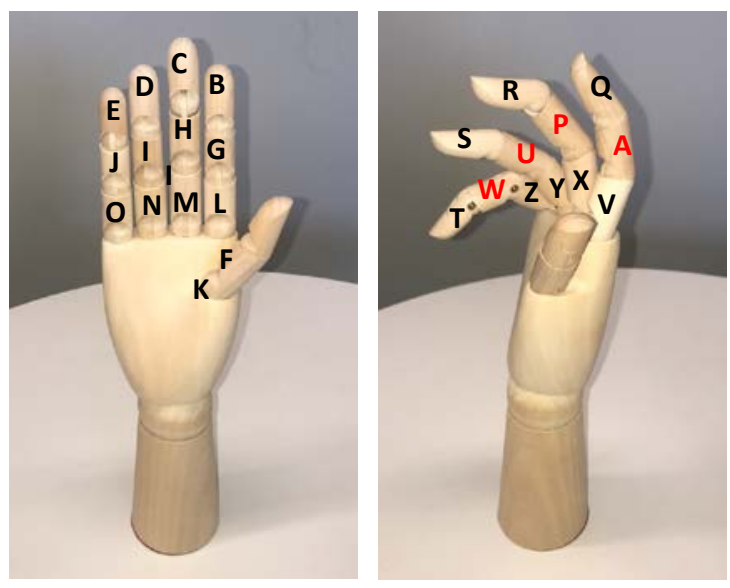

Figure 4. The Mobile Malossi alphabet (adjusted letters are shown in red)

\subsection{Human-robot interactions}

Social robotics is an emerging field with great potential for providing services and assistive support to people with special needs. While the staff of disability service organizations have identified domestic robots and rehabilitation robot applications as primary targets [28], robots that can engage in meaningful social interactions are highly desirable. A socially interactive robot such as Philos [29], for example, can be used for daily health monitoring and emotional stimulation of people in need of continuous care.

Some researchers focus on making social robots more accessible to the deaf [30]. Along this line, social robots have been employed in educational contexts to assist with the learning of sign language for people with hearing disorders [31]. Augmented sign language exposure for deaf infants integrating a robot and a virtual human has also been explored [32].

With respect to the deafblind, the PALORMA system has been devised for support of deafblind remote communications and further enabled with control of robotic hands [33]. A more general tactile communication system specifically designed to support interactions between deafblind and robots is discussed in [34]. Guiding robots may be of particular interest for deafblind people who use guide dogs since such robots are easier to care for [28]. In such situations, the employment of haptic interfaces and motion recognition becomes indispensable for establishing and maintaining a reliable communication channel between the deafblind person and the guiding robot [35].

Our specialized equipment and software for deafblind communications support, presented in the earlier sections, provides for both incremental and qualitative improvements of the current state of the art. While Data Gloves have been employed in a number of earlier works, the advanced nanomaterials research conducted by members of our team has made it possible to produce a novel Data Glove with much different touch and feel. It is thinner, more elastic, and durable and feels like a normal cloth glove which makes it quite suitable for long term wear.

Beginning with the initially produced wired Data Glove, we have made substantial hardware enhancements to allow for local wireless connections and thus enable direct communication with nearby mobile robots. The constructed modular framework incorporates both hardware and software communication components that are specifically designed to enhance the interaction capabilities of deafblind users. We employ the Malossi alphabet which is well known and widely used in the deafblind community. And, with minimal adjustments to the signing process, we have made possible the use of Malossi in truly mobile setouts.

\section{Conclusions and Future Works}

In this paper, we have reviewed some of the traditional communication methods for the deafblind and have considered different possibilities for enhanced support through recent technological achievements. Consequently, we presented our lightweight, and high fidelity, Data Glove, constructed using specialized equipment, including CNT sensors, and software to support deafblind communication. The preliminary experiments with the Data Glove messaging based on the Malossi alphabet have indicated possible signing optimization through minimal, easy-to-remember changes in the letter assignments. We are planning to continue the experimental work with further optimizations of the Malossi-based signing and improvements of the usability of the Data Glove.

\section{Acknowledgment}

This work was partially supported by a Cooperative Research Project at the Research Center 
for Biomedical Engineering and Research Institute of Electronics, Shizuoka University, Hamamatsu, Japan.

\section{References}

[1] Jaiswal, A., Aldersey, H., Wittich, W., Mirza, M., Finlayson, M. (2020). Factors that influence the participation of individuals with deafblindness: A qualitative study with rehabilitation service providers in India. The British Journal of Visual Impairment, 0264619620941886.

[2] Dammeyer, J. (2014). Deafblindness: a review of the literature. Scandinavian Journal of Public Health, 42(7), 554-562.

[3] Caporusso, N., Biasi, L., Cinquepalmi, G., Trotta, G. F., Brunetti, A., Bevilacqua, V. A (2018). Wearable Device Supporting Multiple Touch- and Gesture-Based Languages for the Deaf-Blind. Advances in Human Factors in Wearable Technologies and Game Design, 32-41.

[4] Olson, N., Urbański, J., Krister Persson, N, StarostaSztuczka, J., Fuentes, M. (2019). Sensor Technology, Gamification, Haptic Interfaces in an Assistive Wearable, Journal on Technology and Persons with Disabilities, 80-87.

[5] Jaiswal, A., Aldersey, H. M., Wittich, W., Mirza, M., Finlayson, M. (2018). Participation experiences of people with deafblindness or dual sensory loss: A scoping review of global deafblind literature. PLOS ONE, 13(9), Article e0203772.

[6] Sorgini, F., Caliò, R., Carrozza, M. C. Oddo, C. M. (2018). Haptic-assistive technologies for audition and vision sensory disabilities, Disab. Rehabil., Assistive Technol., vol. 13, no. 4, pp. 394-421.

[7] Goggin, G., Ellis, K. (2020). Disability, communication, and life itself in the COVID-19 pandemic. Health Sociology Review: The Journal of the Health Section of the Australian Sociological Association, 29(2), 168-176.

[8] Caporusso, N. A. (2008). Wearable Malossi alphabet interface for deafblind people. Proceedings of the Working Conference on Advanced Visual Interfaces, 445-448.

[9] Caporusso, N., Biasi, L., Cinquepalmi, G., Trotta, G. F., Brunetti, A., Bevilacqua, V. (2018). Enabling Touch-Based Communication in Wearable Devices for People with Sensory and Multisensory Impairments. Advances in Human Factors in Wearable Technologies and Game Design, 149-159.

[10] Willoughby, L., Iwasaki, S., Bartlett, M. Manns, H. (2018). Tactile sign languages. J. Östman, and J. Verschueren (Eds.), Handbook of Pragmatics, 21st Annual Installment, John Benjamins Publishing Company, pp. 239-258, 2018.

[11] Marschark, M., Spencer, P. E. (2015). The Oxford Handbook of Deaf Studies in Language. Oxford University Press. p. 338.

[12] Gelsomini, F., Kanev, K., Hung, P., Kapralos, B., Jenkin, M., Barneva, R. P., \& Vienna, M. (2017). BYOD Collaborative Kanji Learning in Tangible
Augmented Reality Settings. In D. Luca, L. Sirghi, \& C. Costin (Eds.), Recent Advances in Technology Research and Education (Vol. 660, pp. 315-325). Springer International Publishing.

[13] Barneva, R. P., Gelsomini, F., Kanev, K., Bottoni, P. (2017). Tangible Technology-Enhanced Learning for Improvement of Student Collaboration. Journal of Educational Technology Systems, 0047239517736875.

[14] Kanev, K. (2012). Augmented Tangible Interface Components and Image Based Interactions, International Conference on Computer Systems and Technologies CompSysTech'12, Ruse, Bulgaria, June 22-23, 2012, pp.23-29.

[15] Mellor, C. M., Braille, L. (2006). A Touch of Genius. Boston: National Braille Press.

[16] Foulke, E. (1982) Reading braille. W. Schiff and E. Foulke (Eds.) Tactual perception: A source book. Cambridge University Press, pp. 168-208.

[17] Carrera, A., Alonso, A., De la Rosa, R., Abril, E. J. (2017). Sensing Performance of a Vibrotactile Glove for Deaf-Blind People. NATO Advanced Science Institutes Series E: Applied Sciences, 7(4), 317.

[18] Kramer, J., Leifer, L. (1988). The talking glove. SIGCAPH Comput. Phys. Handicap., 39, 12-16.

[19] Kramer, J. P., Lindener, P., George, W. R. (1991). Communication system for deaf, deaf-blind, or nonvocal individuals using instrumented glove, USPTO Patent No. 5047952, https://patentimages.storage. googleapis.com/26/75/36/c3c9b51f780ed4/US5047952. pdf

[20] Lu, P., Huenerfauth, M. (2009). Accessible motioncapture glove calibration protocol for recording sign language data from deaf subjects. Proceedings of the 11th International ACM SIGACCESS Conference on Computers and Accessibility, 83-90.

[21] Monti, L., Delnevo, G. (2018). On improving GlovePi: Towards a many-to-many communication among deafblind users. 2018 15th IEEE Annual Consumer Communications Networking Conference (CCNC),1-5.

[22] Gollner, U., Bieling, T., Joost, G. (2012). Mobile Lorm Glove: introducing a communication device for deafblind people. Proceedings of the Sixth International Conference on Tangible, Embedded and Embodied Interaction, 127-130.

[23] Mirri, S., Prandi, C., Salomoni, P., Monti, L. (2017). Fitting like a GlovePi: A wearable device for deaf-blind people. 2017 14th IEEE Annual Consumer Communications Networking Conference (CCNC), 1057-1062.

[24] Suzuki, K., Yataka, K., Okumiya, Y., Sakakibara, S., Sako, K., Mimura, H., Inoue, Y. (2016). Rapidresponse, widely stretchable sensor of aligned MWCNT/elastomer composites for human motion detection. Acs Sensors, 1(6), 817-825.

[25] Inoue, Y., Kakihata, K., Hirono, Y., Horie, T., Ishida, A., Mimura, H. (2008). One-step grown aligned bulk carbon nanotubes by chloride mediated chemical vapor deposition. Applied physics letters, 92(21), 213113.

[26] Gelsomini, F., Hung, P. C. K., Kapralos, B., UribeQuevedo, A., Jenkin, M., Tokuhiro, A., Kanev, K., Hosoda, M., Mimura, H. (2021). Specialized CNT- 
based Sensor Framework for Advanced Motion Tracking, The 54th Hawaii Int. Conference on System Sciences HICSS-54. doi: 10.24251/HICSS.2021.231.

[27] Demoe, M., Uribe-Quevedo, A., Salgado, A. L., Mimura, H., Kanev, K., Hung, P. C. K. (2020). Exploring Data Glove and Robotics Hand Exergaming: Lessons Learned, 2020 IEEE 8th International Conference on Serious Games and Applications for Health (SeGAH), Vancouver, BC, Canada, 2020, pp. 18, doi: 10.1109/SeGAH49190.2020.9201747.

[28] Wolbring, G., Yumakulov, S. (2014). Social Robots: Views of Staff of a Disability Service Organization. Int. J. of Soc. Robotics 6, 457-468 (2014). doi: 10.1007/s12369-014-0229-z

[29] Hornfeck, K., Zhang, Y., Lee, K. (2012). Philos: a sociable robot for human robot interactions and wireless health monitoring. In Proceedings of the 27th Annual ACM Symposium on Applied Computing (SAC '12). Association for Computing Machinery, New York, NY, USA, 293-294. doi: 10.1145/2245276.2245334

[30] Yu, H., Lee, H., Bae, J., Kim, M., Choi, S., Hwang, J. (2021). The Development of a Social Robot Accessible to the Deaf. In Companion of the 2021 ACM/IEEE International Conference on Human-Robot Interaction (HRI '21 Companion). Association for Computing Machinery, New York, NY, USA, 634-635. doi: 10.1145/3434074.3446948

[31] Meghdari, A., Alemi, M., Zakipour, M. et al. (2019). Design and Realization of a Sign Language
Educational Humanoid Robot. J Intell Robot Syst 95, 3-17. doi: 10.1007/s10846-018-0860-2

[32] Scassellati, B., Brawer, J., Tsui, K., Gilani, S.N., Malzkuhn, M., Manini, B., Stone, A., Kartheiser, G., Merla, A., Shapiro, A., Traum, D., Petitto, L. (2018). Teaching Language to Deaf Infants with a Robot and a Virtual Human. In Proceedings of the 2018 CHI Conference on Human Factors in Computing Systems (CHI '18). Association for Computing Machinery, New York, NY, USA, Paper 553, 1-13. doi: 10.1145/3173574.3174127

[33] Russo, L. O., Farulla, G. A., Pianu, D., Salgarella, A. R., Controzzi, M., Cipriani, C., Oddo, C. M., Geraci, C., Rosa, S., Indaco, M. (2015). PARLOMA - A Novel Human-Robot Interaction System for Deaf-Blind Remote Communication. International Journal of Advanced Robotic Systems. doi: 10.5772/60416

[34] Ozioko, O. O., Navaraj, W. T., Yogeswaran, N., Hersh, M. and Dahiya, R. (2018). Tactile Communication System for the Interaction between Deafblind and Robots. In:27th IEEE International Symposium on Robot and Human Interactive Communication (ROMAN), Nanjing, China, 27-31 Aug 2018, pp. 416-421. ISBN 9781538679814.

[35] Farulla, G. A., Russo, L. O., Gallifuoco, V., Indaco, M. (2015). A Novel Architectural Pattern to Support the Development of Human-Robot Interaction (HRI) Systems Integrating Haptic Interfaces and Gesture Recognition Algorithms, 2015 IEEE Computer Society Annual Symposium on VLSI, 2015, pp. 386-391, doi: 10.1109/ISVLSI.2015.112. 